\title{
Study on Electromagnetic Wave Propagation Characteristics in Cylindrical Photonic Crystal Waveguides with High-Index Cores
}

\author{
Jeong Kim \\ Dept. of Electric, Electronic and Communication Engineering Education, Chungnam \\ National University, Daejeon 34134, Korea \\ jikimi@cnu.ac.kr
}

\begin{abstract}
Cylindrical photonic crystal waveguides $(C P C W s)$ with high-index cores are first proposed and analyzed by accurate full-vector finite difference method and finite-difference time-domain approach. The high-index core can be realized by inserting materials with higher refractive index values than normal pure-silica glass, for example, carbon dioxide, Liquefied Petroleum Gas ( $L P G)$, and nitrous oxide into the CPCW. It is generally observed that the high refractive index variation is not linearly changing with the normalized propagation constant. More significant discontinuities in field distributions are also noticed at dielectric boundaries for the $C P C W$ with lower refractive index core, compared to the $C P C W$ with higher-index core, which reasonably means that electromagnetic energy is more tightly confined for the $C P C W$ design with higher-index core.
\end{abstract}

Keywords: Photonic band-gap, High-index materials, Cylindrical photonic crystal waveguides, Optical devices

\section{Introduction}

Photonic crystal structures have gained considerable attention from researchers, finding innovational applications as in light-emitting diodes, lasers, nonlinear devices, optical fibers, and photovoltaic cells as a source of power for electronics [1][2][3]. In analogy to the basic concept that a spatial periodic arrangement of atoms or molecules constructs a normal crystal, it has been investigated that a photonic crystal can be formed by changing the relative permittivity of a material with an appropriately repeating pattern in space. Since a photonic crystal possesses a forbidden frequency band, called photonic band-gap, in which propagation of electromagnetic waves is prohibited, light-reflection principle in the photonic crystal structure can produce many of the analogous optical phenomena, such as spontaneous emission control, strong localization of photons, and low-loss waveguiding, akin to the atomic potential acting on electrons [4][5][6]. Thereby, electromagnetic waves are effectively controlled either to propagate through the structure or not, depending on operating wavelengths.

In this paper, Cylindrical Photonic Crystal Waveguides (CPCWs) with high-index cores are first proposed and analyzed by accurate computational methods. Here, the high-index core can be realized by inserting materials with higher refractive index values than normal pure-silica glass, for example, carbon dioxide, Liquefied Petroleum Gas (LPG), and nitrous oxide into the $\mathrm{CPCW}$. Two numerical approaches of the rigorous full-vector Finite-Difference Time-Domain

Article history:

Received (April 25, 2019), Review Result (May 22, 2019), Accepted (June 18, 2019) 
(FDTD) technique and Finite Difference Method (FDM) are considered for cross-verification purpose. Several important propagation characteristics such as normalized propagation constant, chromatic and waveguide dispersion, mode field distribution, and effective area are investigated.

\section{Design and Analysis of the Cylindrical Photonic Crystal Waveguide}

Cylindrically-varying PCWs have periodic index variations only in the azimuthal direction. These waveguides consist of a central core surrounded by a cladding region composed of several alternating-index rings with one equal-thickness repeating pattern. Here, CPCWs with high-index cores are investigated. The refractive index profile for the proposed geometries is shown in [Figure 1]. These optical guides consist of a solid core with a radius of $r 1$, and air and silica-glass layers, of which thicknesses are denoted by da and ds in the refractive index profile, respectively. Therefore, the two da and ds layers together form one repeating period.

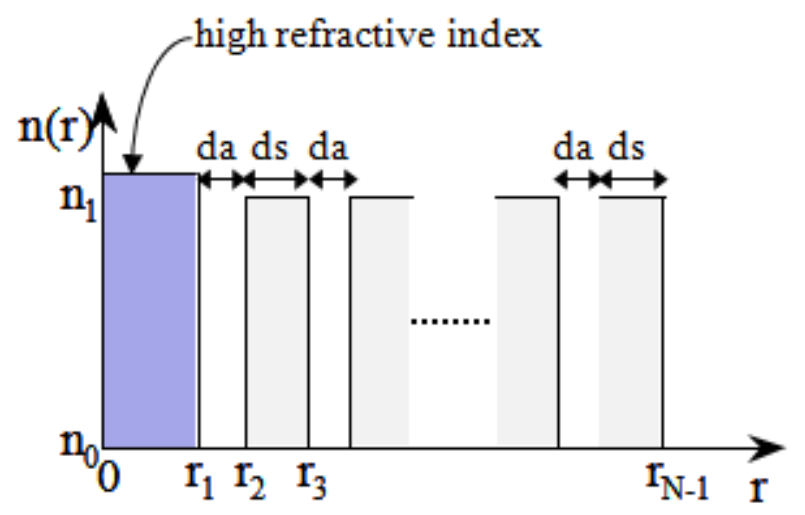

Figure 1. Refractive index profile of a cylindrically-varying PCW with a high-index core

Since the difference between two refractive index values in the periodically-repeating rings of the cladding region can be large, it is necessary to apply a full vectorial approach instead of a scalar-wave approximation to analyze CPCWs. Considering one such light-guiding fiber, which is composed of $\mathrm{N}$ linear, isotropic, homogeneous and cylindrically symmetric dielectric layers as illustrated in [Figure 1], the i-th region, which is filled with a refractive index ni, is defined by an inner radius $r(i-1)$ and an outer radius $r_{i}$, where $i=1,2, \ldots, N$. Because of the periodic index profiles, either a high (silica glass) refractive index or a low (air) refractive index is employed for the ni value. In addition, the core index is designed to be higher than the silicaglass refractive index.

Based on two Maxwell's curl expressions, the proposed CPCWs are investigated by first employing the FDTD method [7]. Considering that the Ex, Ey, Ez, Hx, Hy, and Hz components of electromagnetic fields are inter-related in the rectangular coordinate system, electric and magnetic wave interactions with general three-dimensional objects can be described by six coupled partial differential equations [8].

Furthermore, these six equations are discretized in the space and time domains and used to find field solutions numerically, by assigning a grid point in the rectangular lattice with digitized integers of $1, \mathrm{~m}, \mathrm{n}$, and $\mathrm{v}$ as

$$
[l, m, n]=[l \Delta x, m \Delta y, n \Delta z]
$$


and any function of space and time as

$$
F^{v}(l, m, n)=F(l \Delta x, m \Delta y, n \Delta z, v \Delta t)
$$

where $\Delta \mathrm{x}, \Delta \mathrm{y}$, and $\Delta \mathrm{z}$ are the lattice space increments in the $\mathrm{x}, \mathrm{y}$, and $\mathrm{z}$ coordinate directions, respectively, and $\Delta \mathrm{t}$ is the time increment [9]. By taking central finite difference approximation for space and time derivatives that are accurate to second order, the partial derivatives are expressed as:

$$
\begin{aligned}
& \frac{\partial F^{v}(l, m, n)}{\partial x}=\frac{F^{v}\left(l+\frac{1}{2}, m, n\right)-F^{v}\left(l-\frac{1}{2}, m, n\right)}{\Delta x}+O\left(\Delta x^{2}\right) \\
& \frac{\partial F^{v}(l, m, n)}{\partial t}=\frac{F^{\left(v+\frac{1}{2}\right)}(l, m, n)-F^{\left({ }^{v-\frac{1}{2}}\right)}(l, m, n)}{\Delta t}+O\left(\Delta t^{2}\right) .
\end{aligned}
$$

Along extending the FDTD logic further in the cross-section for the proposed waveguide, infinite electromagnetic media for an arbitrary interesting object need to be modeled carefully. The reason is that although with advanced current technology, computer memory is not limitless in the computation space.

Similar to the FDTD employment, the FDM algorithm is reasonably applied to compute propagation characteristics such as effective refractive index, electromagnetic field profile, and effective mode area [8]. As an initial investigation, CPCWs with $\mathrm{n}_{1}=1.45, \mathrm{r}_{1}=0.5 \mu_{\mathrm{m}}$, da $=$ $0.2 \mu \mathrm{m}, \mathrm{ds}=0.3 \mu \mathrm{m}$, and total 8 layers are designed and analyzed by varying the high refractive index $\left(n_{c}\right)$ of the core to be 1.45, 1.5, and 1.6.

[Figure 2] illustrates the normalized propagation constant versus the operation wavelength $(\lambda)$ for the fundamental mode without taking into account the material dispersion effect [10]. It is noticed that the effective refractive indices for the CPCWs with $\mathrm{n}_{\mathrm{c}}=\mathrm{n}_{1}$ and $\mathrm{n}_{\mathrm{c}}=1.5$ are about 1.284 and 1.312 , respectively, which indicates that the normalized propagation constant is changed by 0.028 at $\lambda=1.3 \mu \mathrm{m}$, while the $\mathrm{n}_{\mathrm{c}}$ value is by 0.05 . By the way, when the $\mathrm{n}_{\mathrm{c}}$ value is changed by 0.1 from 1.5 to 1.6 and the normalized propagation constant is by 0.08 from 1.312 to 1.392 . Therefore, the $\mathrm{n}_{\mathrm{c}}$ value is changed twice from 0.05 to 0.1 , meanwhile the normalized propagation constant is more drastically changed by about 2.86 times.

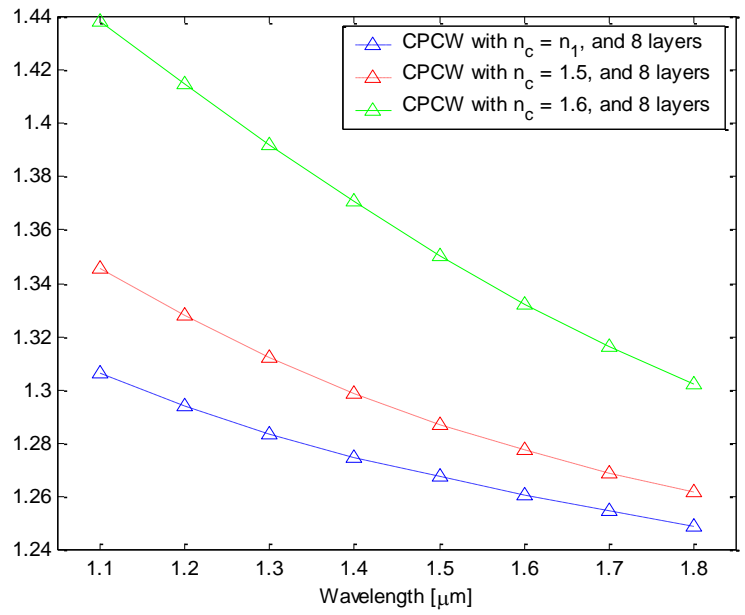

Figure 2. Variations of the effective refractive index versus the operation wavelength for the CPCWs with $\mathrm{r}_{1}=0.5 \mu \mathrm{m}$ 


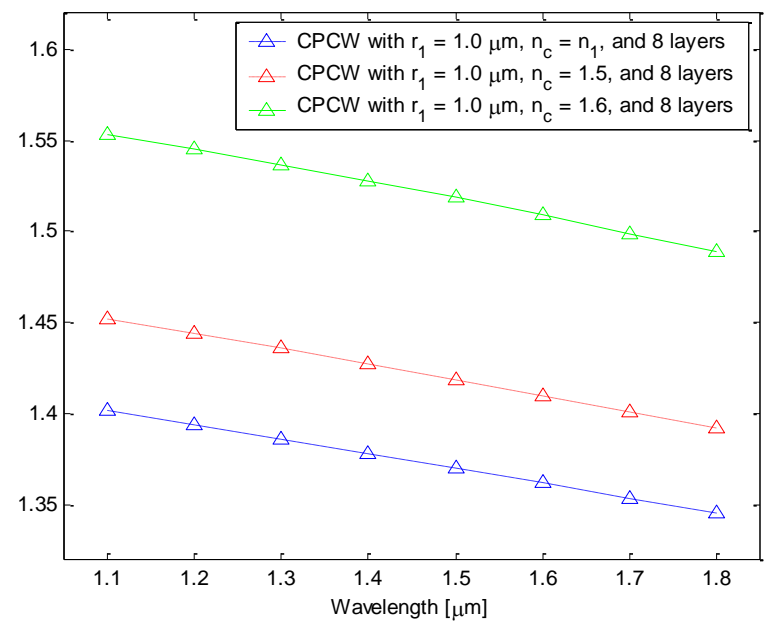

Figure 3. Variations of the effective refractive index versus the operation wavelength for the CPCWs

$$
\text { with } \mathrm{r}_{1}=1.0 \mu \mathrm{m}
$$

For the purpose of extension of comparative investigation, the CPCWs with $\mathrm{n}_{1}=1.45, \mathrm{r}_{1}=$ $1.0 \mu \mathrm{m}, \mathrm{da}=0.2 \mu \mathrm{m}, \mathrm{ds}=0.3 \mu \mathrm{m}$, and total of 8 layers are also designed and analyzed by varying the $\mathrm{n}_{\mathrm{c}}$ value of the core to be $1.45,1.5$, and 1.6. [Figure 3] shows the effective refractive index versus the operating $\lambda$ for the fundamental mode. Now it is noticed that the effective index values vary almost linearly and inversely proportional to the wavelength. And the CPCW with $\mathrm{r}_{1}=1.0 \mu \mathrm{m}$ and $\mathrm{n}_{\mathrm{c}}=1.6$ provides very higher effective index values than 1.55 around $\lambda=$ $1.1 \mu \mathrm{m}$.

During the investigation, electromagnetic field variations are also analyzed. [Figure 4] shows field distributions for the Ex electric components of the fundamental mode at the operation wavelength of $1.3 \mu \mathrm{m}$ for the CPCW designed with $\mathrm{n}_{\mathrm{c}}=\mathrm{n}_{1}$ and $\mathrm{n}_{\mathrm{c}}=1.6$. As expected, more significant discontinuities are noticed at dielectric boundaries for the CPCW with $\mathrm{n}_{\mathrm{c}}=\mathrm{n}_{1}$, compared to the CPCW with $\mathrm{n}_{\mathrm{c}}=1.6$, which means electromagnetic energy is more tightly confined for the CPCW design with $\mathrm{n}_{\mathrm{c}=1}$. .

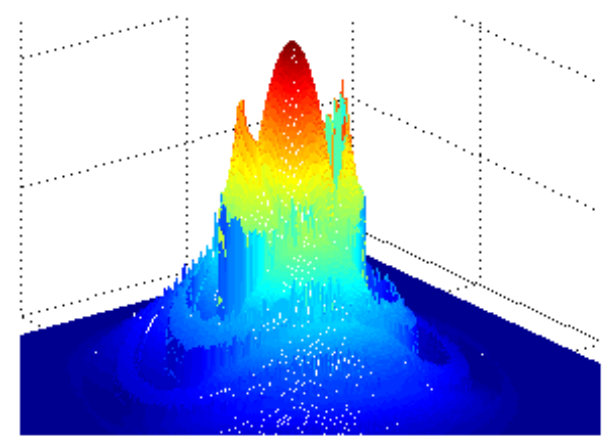

(a)

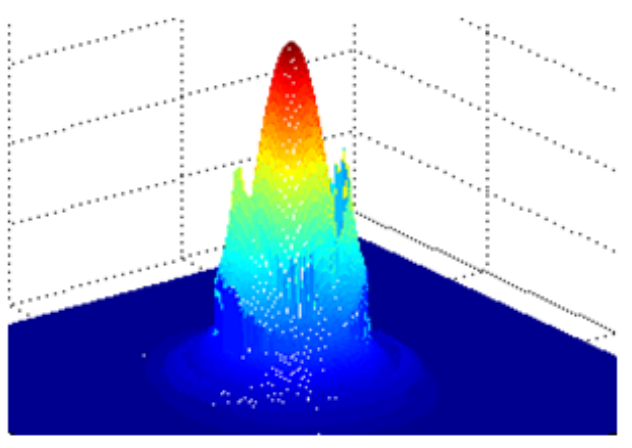

(b)

Figure 4. Ex electric field distributions of the fundamental mode at the operating $\lambda=1.3 \mu \mathrm{m}$ for the CPCW designed with (a) $\mathrm{n}_{\mathrm{c}}=\mathrm{n}_{1}$ and (b) $\mathrm{n}_{\mathrm{c}}=1.6$ 


\section{Conclusion}

CPCWs with high-index cores are first designed and analyzed for accurate full-vector electromagnetic investigation. The high-index core can be realized by inserting materials with higher refractive index values than normal pure-silica glass, for example, carbon dioxide, LPG, and nitrous oxide into the $\mathrm{CPCW}$. In order to firstly see the normalized propagation constant dependency on the core refractive index variation, the material dispersion effect is not taken into account. It is reasonable that more significant discontinuities are noticed at dielectric boundaries for the CPCW with $\mathrm{n}_{\mathrm{c}}=\mathrm{n}_{1}$ at the operating $\lambda=1.3 \mu \mathrm{m}$, compared to the CPCW with $\mathrm{n}_{\mathrm{c}}=1.6$ and total 8 layers, which means electromagnetic energy is more tightly confined for the CPCW designed with $\mathrm{n}_{\mathrm{c}}=1.6$ and the same total layers.

With respect to the normalized propagation constant variations, the result for the CPCW with $\mathrm{n}_{\mathrm{c}}=1.6$ indicates more rapid change, compared to the $\mathrm{CPCW}$ with the lower core index, by increasing the operation wavelengths between $1.1 \mu \mathrm{m}$ and $1.8 \mu \mathrm{m}$. By utilizing the propagation characteristics such as the normalized constant dependency and electromagnetic field variations, inventive applications for optical sensors or nonlinear devices can be expected to be desirably designed.

\section{References}

[1] A. A. Rifat, K. Ahmed, S. Asaduzzaman, B. K. Paul, and R. Ahmed, "Development of Photonic Crystal FiberBased Gas/Chemical Sensors," Computational Photonic Sensors, Springer Nature, Switzerland, (2019)

[2] Md. S. Islam, J. Sultana, A. Dinovitser, K. Ahmed, B. W.-H. Ng, and D. Abbott, "Sensing of toxic chemicals using polarized photonic crystal fiber in the terahertz regime," Optics Communications, vol.426, pp.341-347, (2018) DOI: 10.1016/j.optcom.2018.05.030

[3] D.-K. Hwang, B. Lee, and D.-H. Kim, "Efficiency enhancement in solid dye-sensitized solar cell by threedimensional photonic crystal,” RSC Advances, vol.9, pp.3017-3023, (2013)

[4] E. Yablonovitch, "Inhibited Spontaneous Emission in Solid-State Physics and Electronics," Physical Review Letters, vol.58, no.20, pp.2059-2062 (1987) DOI: 10.1103/PhysRevLett.58.2059

[5] S. John, "Strong localization of photons in certain disordered dielectric superlattices," Physical Review Letters 58, no.23, pp.2486-2489, (1987) DOI: 10.1103/PhysRevLett.58.2486

[6] J. I. Kim, "Investigation of cladding effects on optical guidance of microstructured holey fibres based on FDM and FDTD methods," Journal of Modern Optics, vol.56, no.9, pp.1091-1095, (2009) DOI: $10.1080 / 09500340902944053$

[7] A. Taflove and S. C. Hagness, "Computational Electrodynamics: The Finite-Difference Time-Domain Method," Artech House, Boston, (2005)

[8] J. Kim, "Effects of Core and Cladding on Optical Guidance Properties of Holey Fiber," Optical Communication, InTech, Croatia, (2012) DOI:10.5772/48280

[9] K. S. Kunz and R. J. Luebbers, "The Finite Difference Time Domain Method for Electromagnetics," CRC Press, Boca Raton, (1993)

[10] G. Keiser, "Optical Fiber Communications,” McGraw Hill, New York, (2000)

\section{Authors}

\section{Jeong Kim}

The author is a professor at Chungnam National University. He received his $\mathrm{PhD}$ degree in electrical and computer engineering (ECpE) from Virginia Polytechnic Institute and State 
University in 2003. He worked as a senior engineer at Samsung Electronics Co., Ltd. for about 3 years. His current research interests include convergence engineering, broadband fiber-optic communications, wireless communications, computer-simulation analysis and design of electromagnetic components, and educational statistics. 